\title{
Adenocarcinoma arising within a tailgut cyst: clinicopathological description and follow up of an unusual case
}

\author{
J F Graadt van Roggen, K Welvaart, A de Roos, G J A Offerhaus, P C W Hogendoorn
}

\begin{abstract}
Full clinicopathological details and clinical follow up of a case of malignant transformation within a tailgut cyst are presented. A 43 year old woman presented with signs and symptoms of an imminent threatened abortion. Routine examination identified a coincidental, asymptomatic retrorectal/ presacral mass. Following imaging studies, surgical resection was carried out and an adenocarcinoma arising within a preexistent tailgut cyst was identified by microscopy. Four years later the patient presented with neurological symptoms consistent with local recurrence of the tumour. Surgical biopsies confirmed this diagnosis and she was subsequently started on chemotherapy. She died soon after from a cause unrelated to the disease, after declining further active intervention. Differential diagnosis of such cases includes (cystic) teratoma, epidermal cyst, rectal duplication cyst, anal gland cyst and carcinoma, extension of local carcinoma, and metastatic disease. It is recommended that these lesions be completely excised when detected incidentally.

(F Clin Pathol 1999;52:310-312)
\end{abstract}

Departments

Pathology, Leiden

University Medical

Centre, Building I,

L1-Q, PO Box 9600,

2300RC Leiden, The

Netherlands

J F Graadt van Roggen

P C W Hogendoorn

Department of Radiology, Leiden

University Medical

Centre

A de Roos

Department of Surgery, Leiden University Medical Centre

K Welvaart

Department of Pathology, Amsterdam

Medical Centre, Amsterdam, The

Netherlands

G J A Offerhaus

Correspondence to: Dr Graadt van Roggen. email:

jgraadt@path_1.medfac. leidenuniv.nl

Accepted for publication 27 November 1998
Keywords: adenocarcinoma; tailgut cyst

Tailgut cysts are rare benign lesions occurring in children and adults in the retrorectal/presacral area and represent a persistence of embryological remnants of the postanal gut. ${ }^{12}$ These lesions have been infrequently reported under a variety of synonyms: retrorectal cyst-hamartoma, tail gut vestiges, cyst of postanal intestine, rectal cyst, and myoepithelial hamartoma of the rectum. ${ }^{1}$ Malignant transformation of the epithelial elements has only rarely been reported.

In this report we document an adenocarcinoma arising within a tailgut cyst and discuss the differential diagnosis. The presenting clinicopathological features and subsequent clinical course, including a local recurrence four years after the initial tumour was excised, are also outlined.

\section{Case report}

A 43 year old white woman with no relevant previous medical history of note presented to our gynaecology department with signs and symptoms of an imminent abortion. Routine physical examination and subsequent magnetic resonance imaging (figs 1 and 2) showed a large, asymptomatic, cystic retrorectal/ presacral mass lying free from the colon, gynaecological organs, and sacrum. There were no signs of colonic obstruction and both ovaries were unremarkable. There were no indications for primary malignant disease elsewhere. The patient was referred to our surgical department and radical excision was attempted. Surgery was complicated and at one point cyst leakage occurred; as a consequence extensive lavage was carried out but potential tumour spill could not be excluded.

\section{HISTOLOGY}

The macroscopic specimen comprised a large, well circumscribed and clearly demarcated cystic lesion with a maximum diameter of 13 $\mathrm{cm}$ (fig 3). No contiguous osseous tissue was present. Externally the lesion had a yellow/pink colour with a generally smooth surface; numerous firmer, more solid areas were palpable in the cyst wall. On sectioning, the cut surface revealed a unilocular cystic space containing mucinous, turbid material. The cyst wall was of variable thickness with contiguous solid areas ranging from a few millimetres in diameter to a maximum of $1.5 \mathrm{~cm}$. The luminal cyst lining was smooth but showed scattered foci with a $\mathrm{red} /$ brown granular appearance. A breach in the cyst wall (iatrogenic) was identified. No

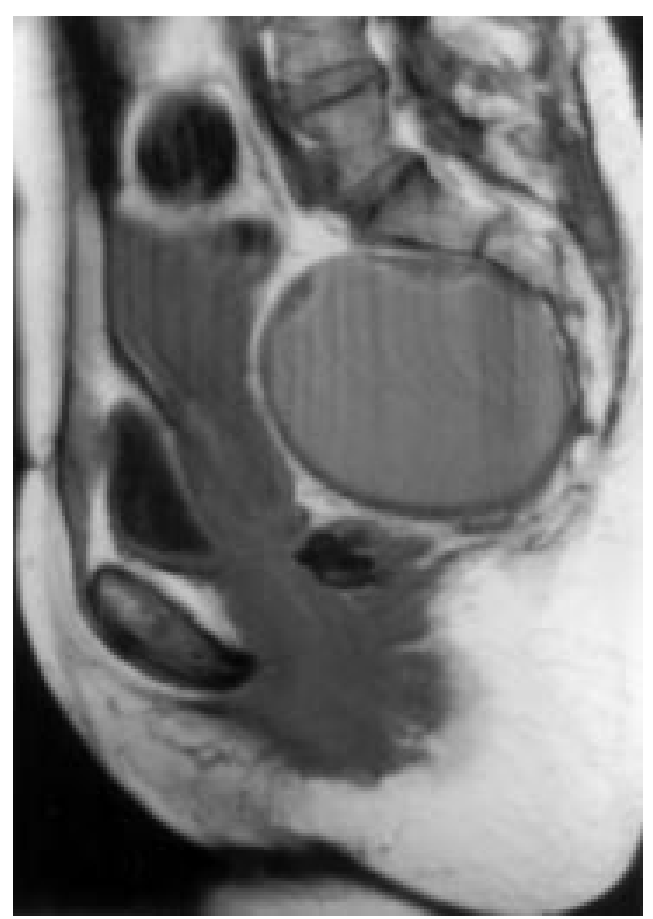

Figure 1 Saggital T1 weighted MRI scan through the pelvis. Note the presacral lesion with intermediate signal intensity. The relation between the mass and the surrounding structures is well depicted. 


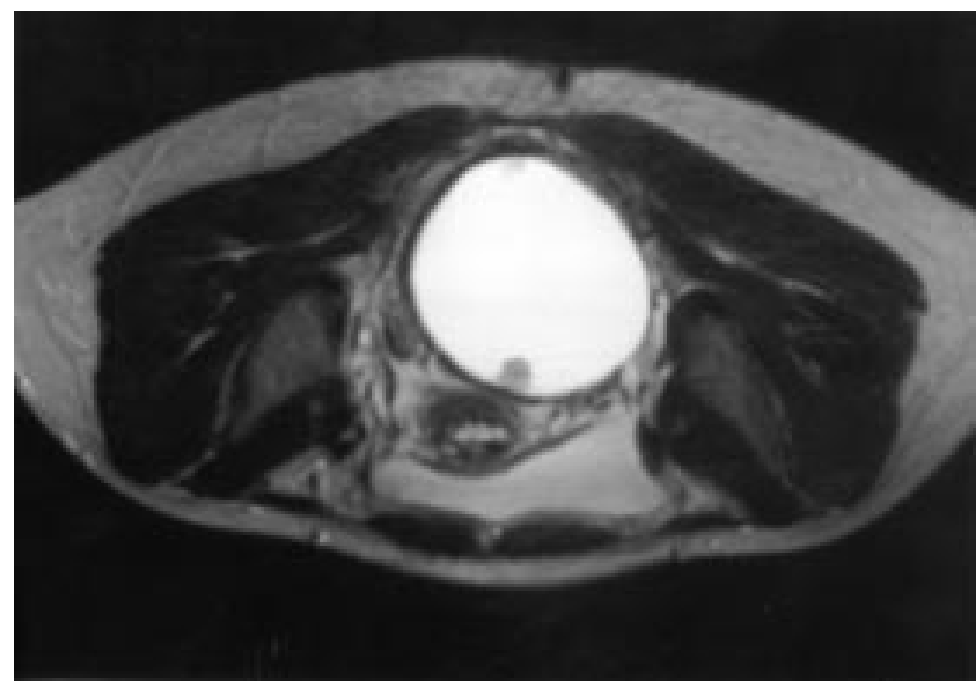

Figure 2 Transverse T2-weighted MRI scan through the pelvis showing a characteristic very high signal intensity of the contents of the lesion, compatible with a cyst-like structure. Note the focal wall thickening anteriorly within the lesion.

hair, cartilage, or other features consistent with teratoma were identified. No obvious necrosis or haemorrhage was present.

Microscopically, multiple sections all showed similar features (figs 4 and 5). The cyst was predominantly lined by tall columnar epithelium containing scattered goblet cells. Isolated foci of ciliated epithelium were noted. Scattered discontinuous bundles of smooth muscle fibres were present in the cyst wall. In addition there were numerous areas of moderately to poorly differentiated adenocarcinoma composed of a solid and tubular growth pattern with abundant mucin. The cells showed prominent nuclear hyperchromasia and pleomorphism, with more than one mitosis per high power field. The resection margins were free of tumour. The alcian blue mucin stain confirmed the presence of the intracellular mucin. The cells were immunohistochemically positive for cytokeratin and carcinoembryonic antigen (CEA); they were negative for the ovarian markers OC-125 and OV-632.

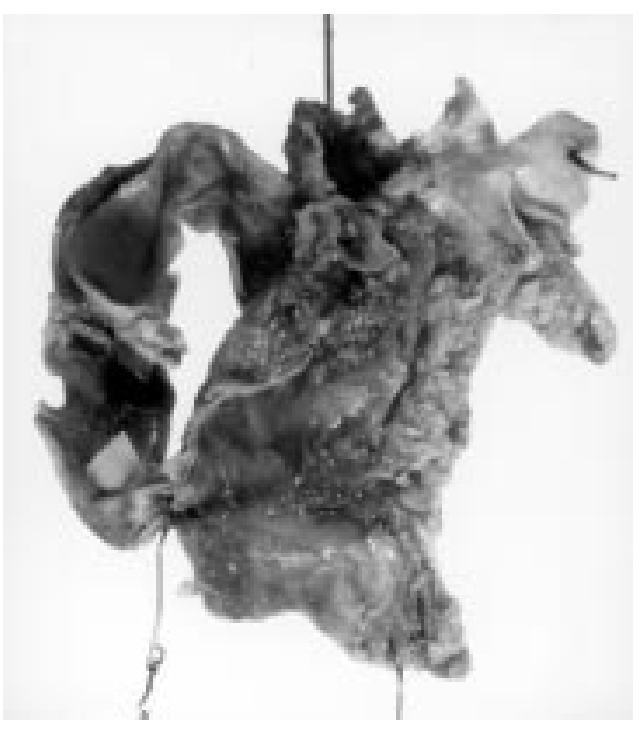

Figure 3 Gross pathology of the resected specimen showing the cystic lesion on cross sectioning.
After considering the differential diagnosismucinous colorectal carcinoma, an epidermal cyst, teratoma, or rectal duplication cyst (all with malignant change), anal gland carcinoma, primary ovarian tumour, metastatic disease to the presacral area, and a tailgut cyst with malignant transformation - a diagnosis of malignant change in a tailgut cyst was made based on the anatomical localisation, histological morphology, cellular architecture, and immunohistochemical profile.

As a result of the intraoperative cyst leakage with potential tumour spill, it was apparent that adjuvent treatment was necessary. Because of the lack of documented information on the management of these cases, and considering the CEA positivity of the tumour cells, it was decided to manage the case along the lines of a CEA positive rectal carcinoma; consequently a total of 50.4 Gy of local radiotherapy was given postoperatively.

Follow up investigations identified no evidence of metastatic disease.

Since the tumour was CEA positive, serum CEA determinations were regularly done to monitor the possibility of tumour recurrence. In the early postoperative period serum CEA remained constant at less than $0.5 \mu \mathrm{g} /$ litre. Three years after the primary resection the patient started complaining of increasing pain in the right buttock; with time this progressed to involve the left buttock and extended down both legs with accompanying weakness, predominantly in an S1 distribution. Additionally, serum CEA began to increase slowly and there was a strong suspicion of a local recurrence involving the sacrum and sacral plexus. Radiological examination, however, could not detect any evidence of sacral, presacral, or local nerve root involvement by tumour. Eventually surgical biopsies, sampling the original surgical site, were carried out and showed extensive local infiltration, including the sacrum, by a moderately to poorly differentiated adenocarcinoma. The histology was compatible with local recurrence of the original tumour. Radiological imaging studies detected no additional metastatic spread. It was decided to start with palliative chemotherapy (5-fluorouracil and Leucovorin (folinic acid)). The patient died soon afterwards from a cause unrelated to the primary disease, after declining further active intervention. Permission for necropsy was not obtained.

\section{Discussion}

The embryological development of the hindgut and anus is such that the most caudal portion of the hindgut (postanal gut) regresses following normal embryogenesis of the anus, rectum, and hindgut. ${ }^{1}$ Persistence of this embryological remnant in the retrorectal/presacral area (bounded anteriorly by the rectum, posteriorly by the sacrum, superiorly by the peritoneal reflection, inferiorly the levators ani and coccygeus muscles, and laterally by the ureters and iliac vessels) is very uncommon and results in the development of a tailgut cyst (retrorectal cyst hamartoma, cyst of postanal intestine, tail gut vestiges, rectal cyst, myoepithelial hamartoma of the rectum) which may present in 

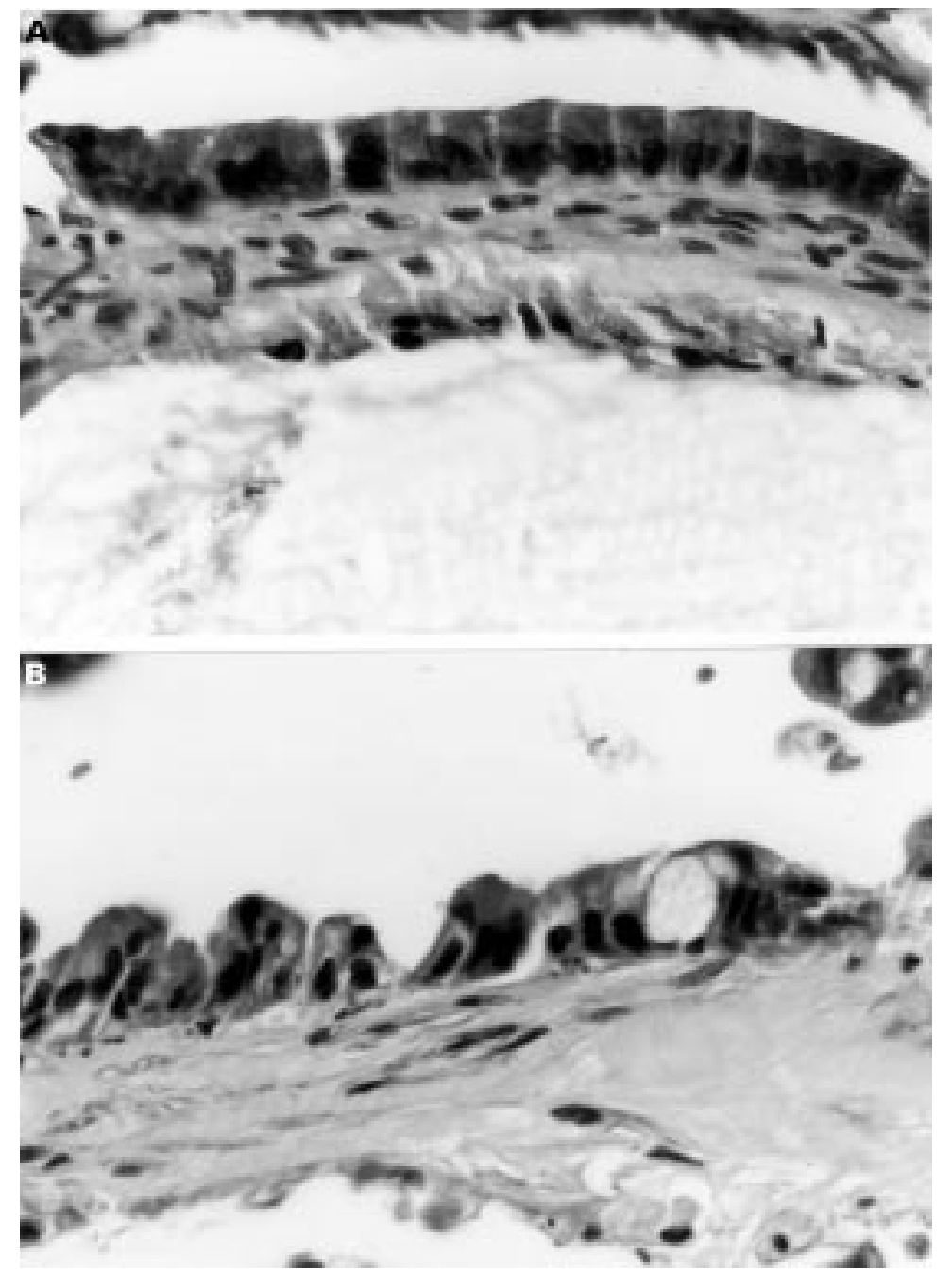

Figure 4 Microscopy of the cyst wall showing the focal, uniform lining of the luminal surface by tall columnar epithelium (A) with scattered goblet cells (B) (×340; haematoxylin and eosin stain).

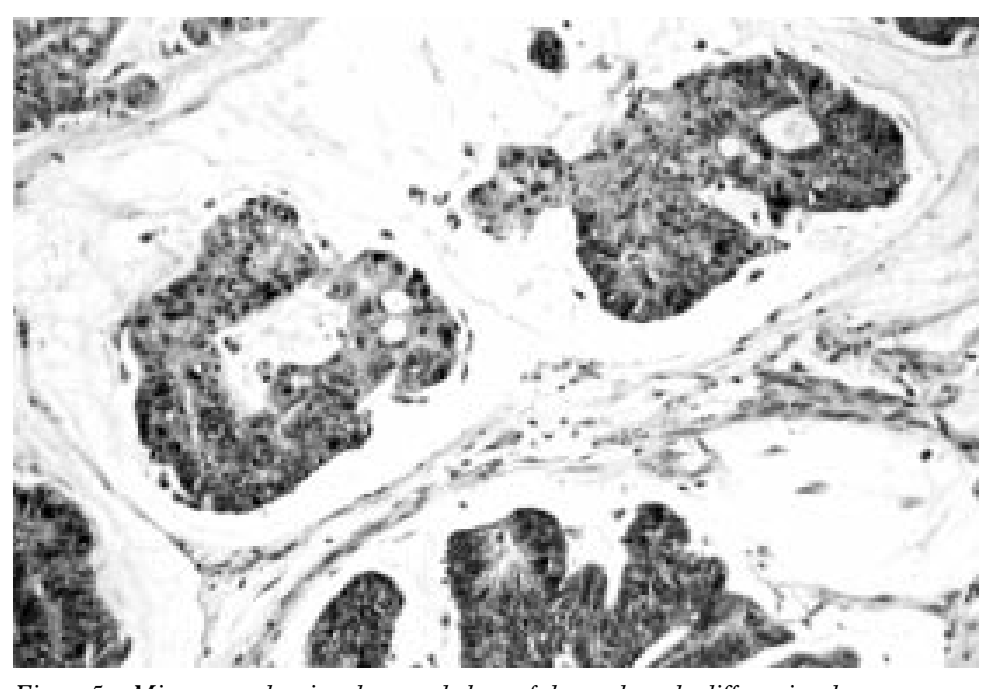

Figure 5 Microscopy showing the morphology of the moderately differentiated adenocarcinoma arising within the tailgut cyst; abundant extracellular mucin production is evident $(\times 170$; haematoxylin and eosin stain).

childhood or adult life with a variety of complications. ${ }^{12}$ The first case of a tailgut cyst with malignant epithelial transformation was described by Ballantine in $1931^{3}$ and since then fewer than 10 cases of this rare phenomenon have been documented in the English literature; the cases described were all adenocarcinomas or carcinoid tumours. ${ }^{1-5}$

According to Peyron's criteria, ${ }^{12}$ tailgut cysts are usually multilocular and are lined by columnar and cuboidal epithelium. Ciliated, squamous, and transitional epithelium has been noted. Acute or chronic inflammation occurs in around $50 \%$ of cases. A muscular and serosal coat is not present, although scattered bundles of smooth muscle fibres may occur in the cyst wall. Most cases are discovered in adulthood and $75-90 \%$ have been described in females. ${ }^{12}$ Tailgut cysts should be distinguished from other lesions which may occur in the retrorectal/presacral space: teratomas, epidermal cysts, rectal duplication cysts, anal gland cysts, and anal gland carcinomas.

Primary ovarian and metastatic disease was excluded in our patient as far as was clinically possible.

Teratomas (and dermoid cysts) have been described in this area and usually comprise components of all three germ layers. The presence of ciliated epithelium in tailgut cysts has been cited to support the concept that the lesions are essentially cystic teratomas. ${ }^{4} \mathrm{Al}-$ though tailgut cysts also contain structures of all three germ layers (epithelium, blood vessels, fibrous tissues, smooth muscle), ciliated epithelium also occurs in the embryo's gastrointestinal tract and is not exclusively of respiratory origin. Hjermstad and Helwig ${ }^{2}$ do not accept the view that the lesions are cystic teratomas in view of their stereotyped histological appearance, cyst organisation, and absence of tissues other than those of the normal fetal gastrointestinal tract.

Epidermal cysts have a different histological morphology, being lined only by squamous epithelium and lacking smooth muscle fibres in their cyst wall.

Rectal duplication cysts differ from tailgut cysts by the presence of a well developed smooth muscle layer.

Anal gland cysts (and anal gland carcinoma) occur in close approximation to the anal sphincter. Although the lining epithelium may be similar in both, tailgut cysts can be distinguished from the former by their anatomical location in the retrorectal space.

Many patients with tailgut cysts probably remain asymptomatic. Tailgut cysts, however, may grow to considerable size and present with gynaecological and obstructive symptoms, chronic abscesses and fistulas, and in extremely rare cases with malignant transformation, as in our case. For this reason, if discovered, complete surgical resection should be considered.

1 Marco V, Autonell J, Farre J, et al. Retrorectal cysthamartomas. Report of two cases with adenocarcinoma developing in one. Am $\mathcal{F}$ Surg Pathol 1982;6:707-14.

2 Hjermstad BM, Helwig EB. Tailgut cysts. Report of 53 cases. Am f Clin Pathol 1988;89:139-47.

3 Ballantine EN. Sacrococcygeal tumours. Adenocarcinoma of a cystic congenital embryonal remnant. Arch Pathol 1931;14:1-9.

4 Uhlig BE, Johnson RL. Presacral tumours and cysts in adults. Dis Colon Rectum 1975;18:581-96.

5 Rosai J, ed. Ackerman's surgical pathology, 8th ed. New York: CV Mosby, 1995. 